\title{
New Records of Two Stichotrichid Ciliates, Afroamphisiella multinucleata and Pseudokahliella marina (Ciliophora: Spirotrichea: Stichotrichida) from Korea
}

\author{
Jung Min Choi ${ }^{1,2}$, Mann Kyoon Shin ${ }^{1, *}$ \\ 'Department of Biological Science, University of Ulsan, Ulsan 680-749, Korea \\ ${ }^{2}$ South Sea Environment Research Division, Korea Institute of Ocean Science and \\ Technology, Geoje 656-830, Korea
}

\begin{abstract}
Two stichotrichid ciliates, collected from marine waters in Jeju Island, were identified as Afroamphisiella multinucleata Foissner et al., 2002 and Pseudokahliella marina (Foissner et al., 1982) Berger et al., 1985. They are recorded for the first time in Korea. The descriptions are based on examinations of living as well as protargol-impregnated specimens. These species are characterized as follows. Afroamphisiella multinucleata has a body size in vivo of 70-95 $\times 20-35 \mu \mathrm{m}$; elongate rectangular in shape; contractile vacuole located slightly above mid-body. The adoral zone is bipartited into 3 distal and 13-17 proximal membranelles and occupies $28-35 \%$ of the body length. The frontal row comprises 1-4 cirri and one buccal cirrus. The amphisiellid median cirral row is composed of 14-21 cirri, 10-19 left marginal cirri, and 21-30 right marginal cirri. Cortical granules are yellowish. 11-20 globular/ellipsoidal macronuclear nodules arrange proximally along the cell margins. Pseudokahliella marina has a body size in vivo of 110-195 $\times 40-110 \mu \mathrm{m}$ and broadly elliptical in shape. The adoral zone of the membranelles occupies 50-60\% of the body length, and is composed of 41-70 membranelles. A prominent frontal scutum is present. The contractile vacuole is located below the mid-body. There are 1113 frontoventral rows, including marginal rows. Caudal cirri and transverse cirri are absent. Three invariable non-fragmented bipolar dorsal kineties are present. The left and right marginal rows are composed of 22-35 and 28-40 cirri, respectively. Colourless cortical granules are present. 8-11 spherical/ellipsoidal macronuclear nodules are connected with each other by thread-like tructures, forming an inverted $\mathrm{C}$-shape.
\end{abstract}

Keywords: Afroamphisiella, Pseudokahliella, hypotrichs, morphology, marine, Jeju Island, Korea

\section{INTRODUCTION}

The order Stichotrichida was established by Fauré-Fremiet (1961) on the bases of having a generally ellipsoidal body shape, being small to large in size, and having one or more longitudinally-arranged ventral cirral row. This order includes six families (Corliss, 1979; Berger, 1999, 2006, 2008, 2011; Lynn and Small, 2002; Lynn, 2008). The two stichotrichs in present study belong to the families Amphisiellidae and Kahliellidae. These two families are quite different from each other in their morphology and morphogenesis. The members of Amphisiellidae have a single ventral cirral row formed by two segments comprising ventral anlage IV and V, whereas more than two ventral cirral rows and neokinetal anlage are present in those of Kahliellidae. The amphisiellid genus Afroamphisiella which includes two species can be distinguished from other amphisiellid genera by the short rows located at the left side of the amphisiellid median cirral row, and the lack of caudal and transverse cirri (Foissner et al., 2002; Berger, 2008). The kahliellid genus Pseudokahliella is monotypic and differs from other kahliellid genera by the absence of parental cirral row and dorsomarginal kineties (Foissner et al., 1982). The morphological descriptions on these species were rare and still poor to define their exact status so far. In this study, we provide alternative morphological descriptions for two stichotrichous ciliates collected from Korean coastal waters and demonstrate their variations from previous descriptions by comparisons of relevant popu-

\footnotetext{
(C) This is an Open Access article distributed under the terms of the Creative Commons Attribution Non-Commercial License (http://creativecommons.org/ licenses/by-nc/3.0/) which permits unrestricted non-commercial use, distribution, and reproduction in any medium, provided the original work is properly cited.

pISSN 2234-6953 eISSN 2234-8190
}

*To whom correspondence should be addressed

Tel: 82-52-259-2396, Fax: 82-52-259-1694

E-mail: mkshin@ulsan.ac.kr 
lations.

\section{MATERIALS AND METHODS}

\section{Sample collection and enrichment}

Ciliates samples were collected from marine waters in the tidal pool on the basal rock in Gwangchigi coast (33 $27^{\prime} 04^{\prime \prime}$ $\left.\mathrm{N}, 126^{\circ} 55^{\prime} 28^{\prime \prime} \mathrm{E}\right)$ located in Jeju Island: Afroamphisiella multinucleata (January, 2010; salinity 36\%o) and Pseudokahliella marina (September, 2010; salinity 31\%o). The specimens were then transferred either to a Petri dish $(87 \mathrm{~mm}$ in diameter) or to a cell culture dish (150 $\mathrm{mm}$ in diameter). These ciliates were cultured at room temperature in the laboratory with the collected water, and dried wheat grain were used to enrich fungi and bacteria as food resources for the ciliates (Lee et al., 1985; Shin, 1994).

\section{Morphological observation}

The morphology of the living specimens was examined using a light microscope equipped with a DIC device (Axio Imager A1; Carl Zeiss, Oberkochen, Germany) at low (50-400 $\times$ ) and high $(1,000 \times$; immersion oil $)$ magnifications. The images of specimens were captured by a CCD camera (Axio Cam MRc; Carl Zeiss). The infraciliatures were examined after protargol impregnations (Wilbert, 1975; Foissner, 1992). The terminology and the taxonomic classification used follow the guidelines given by Berger (2008, 2011) and Lynn (2008).

\section{SYSTEMATIC ACCOUNTS}

Order Stichotrichida Fauré-Fremiet, 1961

Family Amphisiellidae Jankowski, 1979

1*Genus Afroamphisiella Foissner, Agatha and Berger, 2002

\section{2*Afroamphisiella multinucleata Foissner, Agatha and} Berger, 2002 (Tables 1, 2, Figs. 1-3)

Uroleptoides kihni: Borror and Evans, 1979: 54.

Afroamphisiella multinucleata Foissner et al., 2002: 699; Berger, 2008: 372

Description. General morphology and behavior: Body size $70-95 \times 20-35 \mu \mathrm{m}$ in vivo, usually about $80 \times 25 \mu \mathrm{m}$; length:width ratio $2.8-4.3: 1$, on average $3.3: 1$. Body shape elongate rectangular; both ends broadly rounded; anterior portion usually curved leftwards (Figs. 1A, 2B, C); dorsoventrally flattened approximately 1.4:1 (Figs. 1D, 2D). Single contractile vacuole sometimes with two collecting canals, slightly above of the mid-body and near left cell margin (Figs. 1A, B, 2A, F). Body very flexible but not contractile (Fig. 2E). Cytoplasm mainly colourless at low magnification, including some fatty shining globules (Fig. 2J). Locomotion usually crawling on substrate, bending to change the direction.

Buccal field and oral infraciliature: Adoral zone of membranelles bipartited into proximal and distal portion by distinct gap (Figs. 1A, F, 3A), occupied $28-35 \%$ of body length and composed of 16-20 adoral membranelles (Table 1, Figs. $1 \mathrm{~F}, 3 \mathrm{~A})$. Buccal area narrow and shallow, posterior half covered by projecting buccal lip (Fig. 1A). Paroral and endoral membranes parallel, endoral longer than paroral (Figs. 1F, 3E). Pharyngeal fibres distinct after protargol impregnation, about $20 \mu \mathrm{m}$ (Figs. 1F, 3A)

Somatic infraciliature: About 3-4, usually 3 frontal cirri (Figs. 1F, 3C). One buccal cirrus located at anterior end of endoral membrane (Figs. 1F, 3E). One row of cirri longitudinally arranged (frontal-row cirri) (Figs. 1F, 3D) below rightmost frontal cirrus, composed of 1-4, usually three cirri. Amphisiellid median cirral row commenced slightly behind rightmost frontal cirrus, comprising 14-21 cirri. Right marginal row with 21-30 cirri, extending posterior end (Fig. 3F), left marginal row with 10-19 cirri (Figs. 1F, 3A). Caudal cirri and transvers cirri absent. Dorsal bristles 2-4 $\mu \mathrm{m}$ long (Figs. $1 \mathrm{G}, 2 \mathrm{H})$. Two to three dorsal kineties extending to posterior portion, rarely third short row present (Figs. 1G, 3B).

Cortical granules: Cortical granules spherical in shape, longitudinally arranged on both ventral and dorsal sides, slightly to obviously yellow in colour, about $0.5 \mu \mathrm{m}$ in diameter (Figs. 1B, C, E, 2I).

Nuclear apparatus: 11-20 globular to ellipsoidal macronuclear nodules, mostly arranged along cell margins, 3-10 $\mu \mathrm{m}$ in length. Two to five micronuclei, spherical, about $2 \mu \mathrm{m}$ in diameter (Figs. 1G, 3A)

Distribution. Africa (Namibia), Asia (Korea [present study]), and North America(USA).

Remarks. The genus Afroamphisiella was established based on the African population by Foissner et al. (2002). Only two species, Afroamphisiella multinucleata and A. abdita, have been known so far in this genus. According to a review by Berger (2008), the Uroleptoides kihni Wenzel, 1953 sensu Borror and Evans (1979) was a misidentification of A. multinucleata. We also consent to this opinion because its nuclear apparatus and cirral pattern are very similar to A. multinucleata.

The Korean population of $A$. multinucleata closely matched the original description of Foissner et al. (2002) in major aspects of morphology. However, the Korean population is

Korean name: ${ }^{1 *}$ 아프리카양열 하모충속, ${ }^{2 *}$ 다핵 아프리카양열하모충 

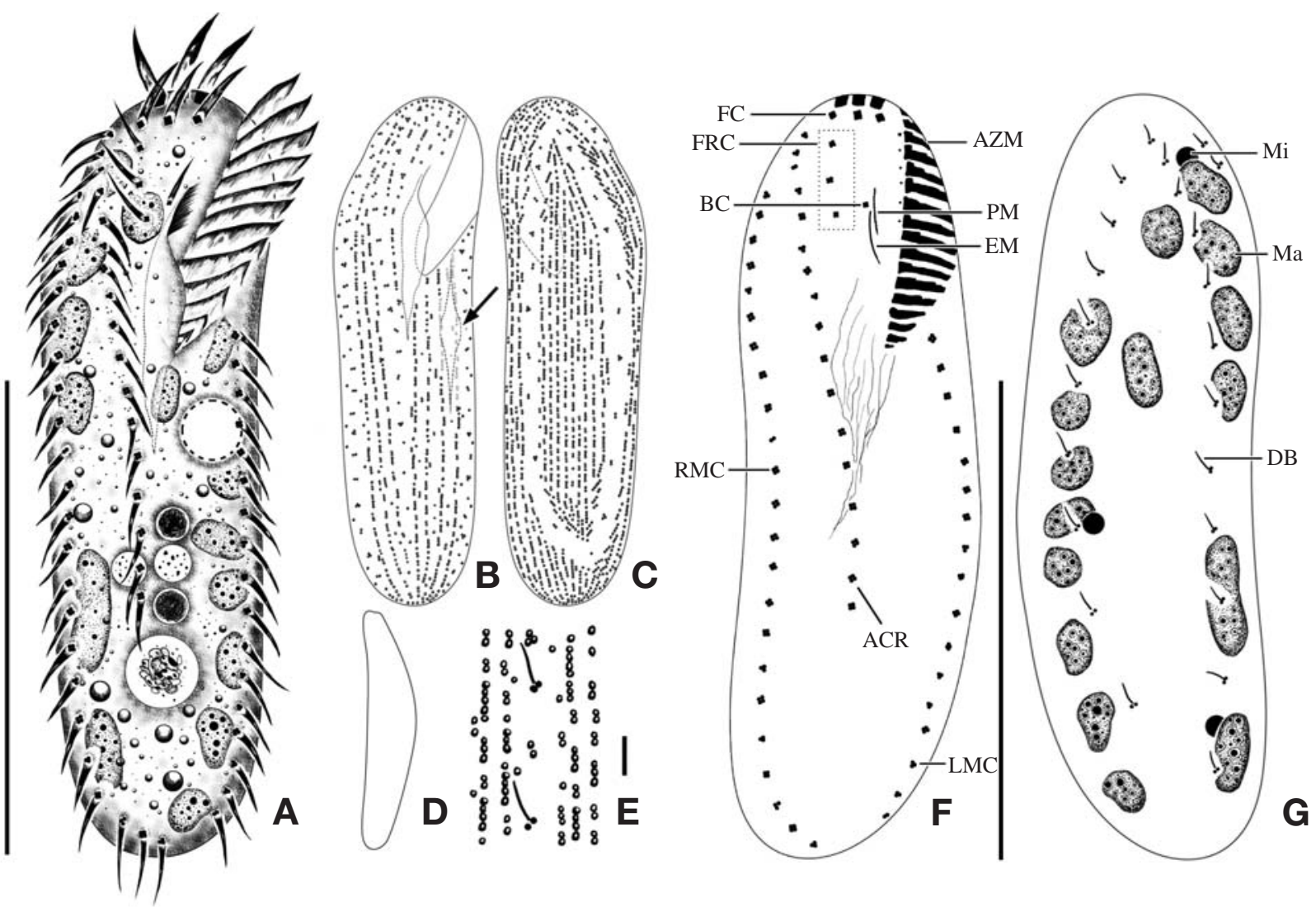

Fig. 1. Afroamphisiella multinucleata from live $(A-E)$ and protargol impregnated specimens $(F, G)$. A, Ventral view of typical cell; $B$, C, Arrangement of canals (arrow) and cortical granules on ventral and dorsal sides; D, Flattened lateral view; $E$, Cortical granules on dorsal side; $F$, Somatic and oral infraciliature of ventral side; G, Dorsal kineties and nuclear apparatus. ACR, amphisiellid median cirral row; $\mathrm{A}(\mathrm{Z}) \mathrm{M}$, adoral (zone of) membranelles; $\mathrm{BC}$, buccal cirrus; $\mathrm{DB}$, dorsal bristles; $\mathrm{EM}$, endoral membrane; $\mathrm{FC}$, frontal cirri; FRC, frontotal-row cirri (dotted box); LMC, left marginal cirri; Ma, macronuclei; Mi, micronuclei; PM, paroral membrane; RMC, right marginal cirri. Scale bars: $A, G=50 \mu m, E=5 \mu m$.

slightly different from the African population in the length of the right marginal row. In the Korean population, the right marginal row usually extends to the posterior end of cell, whereas in the African population, it does not. The shape of the cortical granules is slightly different in both Korean and the African populations (spherical vs. ellipsoidal). The Korean population of A. multinucleata is different from the other congener, A. abdita, in the number of macronuclear nodules (11-20 vs. 2-4), the number of buccal cirri (constantly 1 vs. 1-2), the number of left marginal cirri (10-19 vs. 22-34), the short rows between frontal row cirri and amphisiellid median cirral row (absent vs. present), and in the cortical granules (present vs. absent) (Foissner et al., 2002; Berger, 2008) (Table 2).

\footnotetext{
${ }^{1 *}$ Family Kahliellidae Tuffrau, 1979
}

\footnotetext{
2*Genus Pseudokahliella Berger, Foissner, and Adam, 1985
}

\section{${ }^{3 *}$ Pseudokahliella marina (Foissner, Adam and Foissner, 1982) Berger, Foissner and Adam, 1985 (Tables 3, 4,} Figs. 4-6)

Kahliella marina Foissner et al., 1982: 218.

Pseudokahliella marina: Berger et al., 1985: 309; Berger, 2001: 41; $\mathrm{Hu}$ and Song, 2003: 2033; Song et al., 2003: 161; Song et al., 2009: 398; Berger, 2011: 663.

Description. General morphology and behavior: Body size $110-195 \times 40-110 \mu \mathrm{m}$ in vivo, usually about $160 \times 77 \mu \mathrm{m}$; length:width ratio about $2.2: 1$. Body shape usually broadly elliptical with both ends rounded but rarely elongated elliptical in shape (Figs. 4A, C, 5A, C); dorsoventrally flattened in 

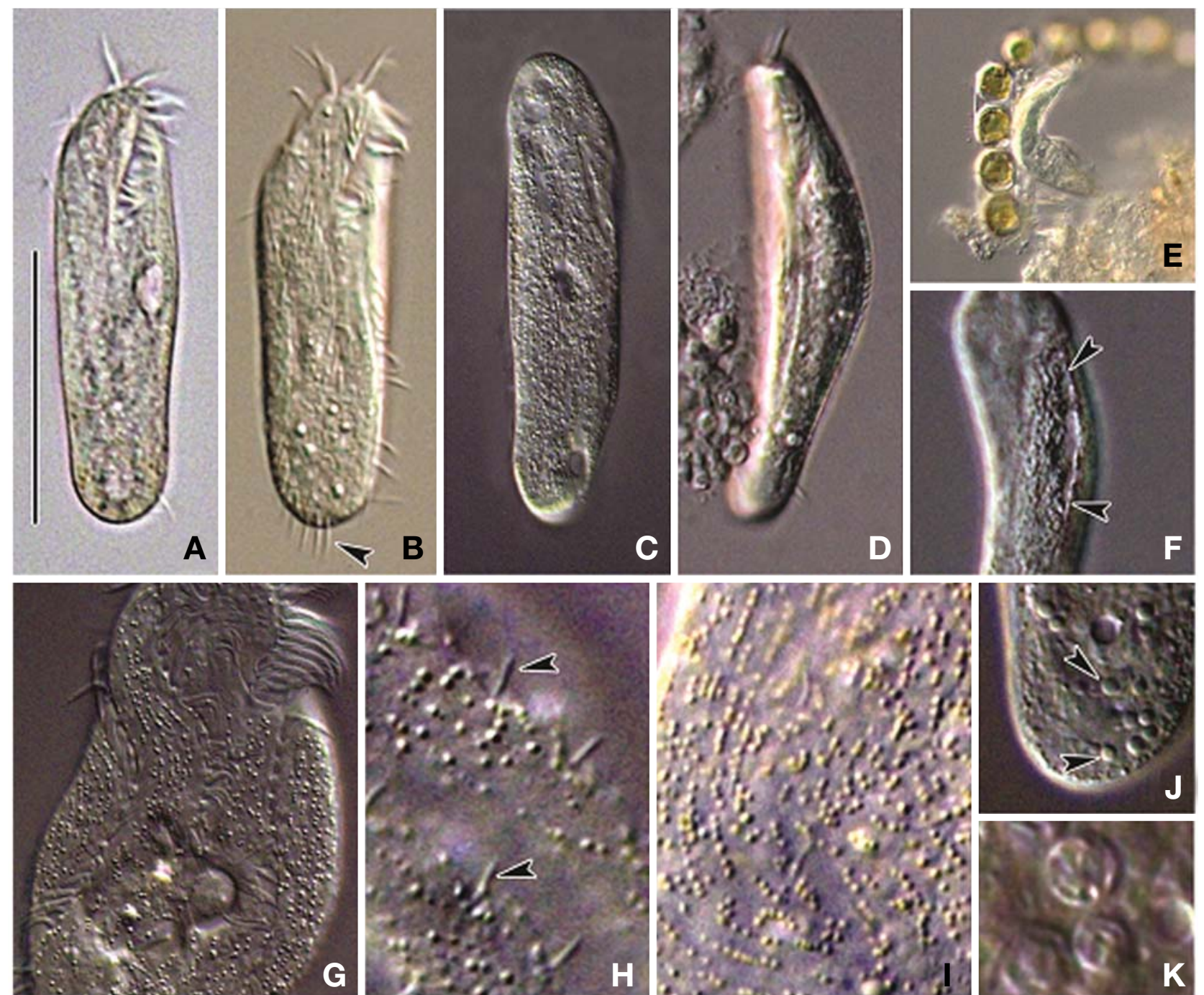

Fig. 2. Photomicrographs of Afroamphisiella multinucleata from live specimens. A, Ventral view of typical cell; B, Somatic ciliature and end of right marginal row (arrowhead) on ventral side; C, Arrangement of cortical granules on dorsal side; D, Flattened lateral view; E, Showing flexible body; F, Contractile vacuole with collecting canal (arrowheads); G, Arrangement of cortical granules on ventral side; $\mathrm{H}$, Dorsal bristles (arrowheads); I, Patterns of cortical granules on dorsal side; J, Fat granules of posterior part (arrowheads); K, Food vacuoles. Scale bar: $A=50 \mu \mathrm{m}$.

an approximately $3: 1$ ratio; ventral side slightly concave; dorsal side distinctly convex (Figs. 4B, 5D, E). Prominent frontal scutum on anterior end of cell (Figs. 4A, C, 5B). Body very flexible but not contractile. Cytoplasm almost colourless at low magnification, including greenish shining globules and yellowish crystals (Fig. 5L-N). Single contractile vacuole spherical in shape, located below mid-body and slightly rightwards, size about $20 \mu \mathrm{m}$ in diameter (Figs. 4A, 5F). Locomotion usually crawling on substrate and swimming spirally. Omnivorous feeding (Figs. 5K, 6J, K).

Buccal field and oral infraciliature: Adoral zone of membranelles occupied $50-60 \%$ of body length (Figs. 4F, 6A), continuous and semicircular, distal end extended to dorsal side of anterior end (Figs. 4F, G, 6B), composed of 41-70 adoral membranelles (Table 3, Figs. 4F, 6E), bases of widest membranelle about $13 \mu \mathrm{m}$ in length. Buccal cavity narrow and rather deep (Figs. 4A, 5B, C). Buccal lip prominent (Figs. 4A, C, 5G). Undulating membranes long, straight to slightly curved (Figs. 4F, 6A). Paroral extended to near proximal end of adoral, about $60 \mu \mathrm{m}$ long; endoral close to paroral, about $40 \mu \mathrm{m}$ long (Figs. 4F, 6E). Pharyngeal fibres about $35 \mu \mathrm{m}$ long and extended transversally (Figs. 4F, 5H, 6A).

Somatic infraciliature: Constantly three frontal cirri, near anterior end region, two slightly enlarged cirri connected to 

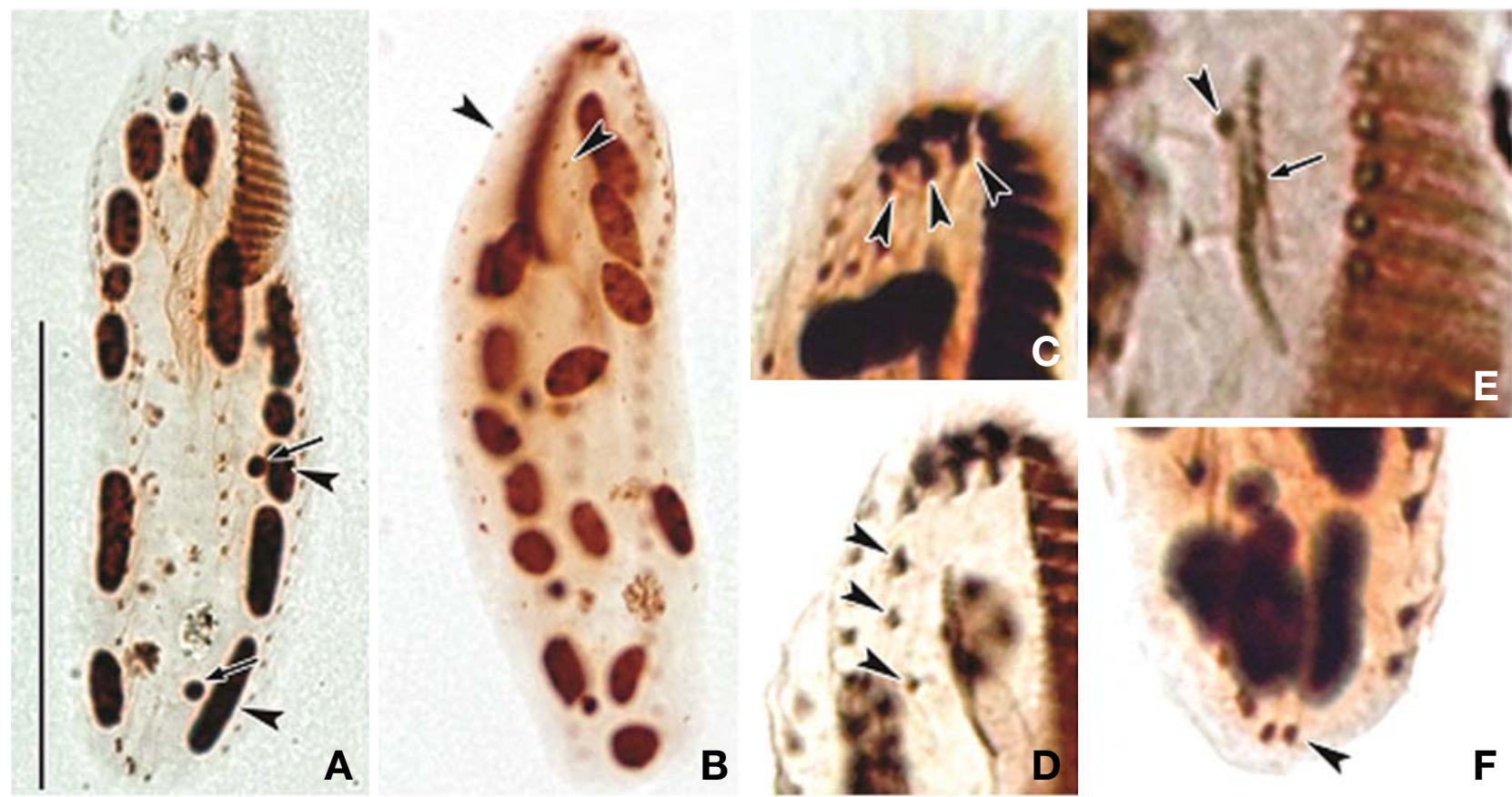

Fig. 3. Photomicrographs of Afroamphisiella multinucleata after protargol impregnation. A, Ventral view of typical cell to indicate macronuclei (arrowheads) and micronuclei (arrows); B, Dorsal view to indicate dorsal kineties (arrowheads); C, Three frontal cirri (arrowheads); D, Frontal row cirri (arrowheads); E, Buccal cirrus (arrowhead) and undulating membranes (arrow); F, Right marginal row extending to posterior end of cell (arrowhead). Scale bar: $A=50 \mu \mathrm{m}$.

Table 1. Morphometric data of Afroamphisiella multinucleata

\begin{tabular}{|c|c|c|c|c|c|c|c|c|}
\hline Characters & Mean & Min & Max & Med & SD & SE & $\mathrm{CV}$ & $\mathrm{n}$ \\
\hline Body, length $(\mu \mathrm{m})$ & 80.3 & 65 & 94 & 80.2 & 8.24 & 1.89 & 10.25 & 19 \\
\hline Body, width $(\mu \mathrm{m})$ & 25.0 & 17 & 31 & 25.6 & 4.06 & 0.93 & 16.21 & 19 \\
\hline Body, length/width & 3.2 & 2.8 & 4.2 & 3.0 & 0.47 & 0.11 & 14.33 & 19 \\
\hline AZM, length $(\mu \mathrm{m})$ & 27.2 & 24 & 29 & 27.4 & 1.45 & 0.33 & 5.34 & 19 \\
\hline Body, length/AZM, length & 2.9 & 2.3 & 3.3 & 3.0 & 0.26 & 0.06 & 8.73 & 19 \\
\hline Ma, number & 15.5 & 11 & 20 & 15.5 & 2.19 & 0.49 & 14.16 & 20 \\
\hline Mi, number & 3.2 & 2 & 5 & 3 & 0.93 & 0.21 & 29.63 & 20 \\
\hline Distal AMs, number & 3.0 & 3 & 3 & 3 & 0.00 & 0.00 & 0.00 & 20 \\
\hline Proximal AMs, number & 15.0 & 13 & 17 & 15 & 1.10 & 0.25 & 7.35 & 20 \\
\hline AMs, number & 18.0 & 16 & 20 & 18 & 1.10 & 0.25 & 6.12 & 20 \\
\hline FC, number & 3.2 & 3 & 4 & 3 & 0.37 & 0.08 & 11.63 & 20 \\
\hline $\mathrm{BC}$, number & 1.0 & 1 & 1 & 1 & 0.00 & 0.00 & 0.00 & 18 \\
\hline FRC, number & 2.7 & 1 & 4 & 3 & 0.81 & 0.18 & 30.67 & 20 \\
\hline ACR, number & 16.9 & 14 & 21 & 17 & 1.65 & 0.40 & 9.80 & 17 \\
\hline LMC, number & 15.6 & 10 & 19 & 16 & 2.21 & 0.54 & 14.17 & 17 \\
\hline RMC, number & 24.2 & 21 & 30 & 24 & 2.27 & 0.52 & 9.39 & 19 \\
\hline DKs, number & 2.2 & 2 & 3 & 2 & 0.41 & 0.11 & 18.82 & 15 \\
\hline DK1, number & 9.2 & 6 & 14 & 9 & 2.14 & 0.53 & 23.25 & 16 \\
\hline DK2, number & 11.1 & 9 & 13 & 12 & 1.41 & 0.36 & 12.64 & 15 \\
\hline DK3, number & 2.0 & 1 & 3 & 2 & 0.82 & 0.41 & 40.82 & 4 \\
\hline
\end{tabular}

All data are based on protargol-impregnated specimens.

Min, minimum; Max, maximum; Med, median; SD, standard deviation; SE, standard error; CV, coefficient of variation in \%; $n$, number of cells; $A(Z) M$, adoral (zone of) membranelles; Ma, macronuclei; Mi, micronuclei; FC, frontal cirri; BC, buccal cirrus; FRC, frontotal-row cirri; ACR, amphisiellid median cirral row; LMC, left marginal cirri; RMC, right marginal cirri; DK, dorsal kineties. 
Table 2. Comparisons between different populations of Afroamphisiella multinucleata and $A$. abdita

\begin{tabular}{lcccc}
\hline \multicolumn{1}{c}{ Characters } & A. multinucleata & A. multinucleata & A. multinucleata & A. abdita \\
\hline Body, length $(\mu \mathrm{m})$ & $65-94$ & $59-84$ & 53 & $80-117$ \\
Body, width $(\mu \mathrm{m})$ & $17-31$ & $18-29$ & 15 & $24-34$ \\
Distal AMs, number & Constantly & $3-4$ & 3 & - \\
Proximal AMs, number & $13-17$ & $13-16$ & 15 & - \\
Total AMs, number & $16-20$ & $16-19$ & 18 & $18-22$ \\
BC, number & Constantly & Constantly 1 & 2 & $1-2$ \\
FRC, number & $1-4$ & $1-3$ & 17 & $2-3$ \\
ACR, number & $14-21$ & $11-22$ & 17 & $14-21$ \\
LMC, number & $10-19$ & $11-22$ & 25 & $22-34$ \\
RMC, number & $21-30$ & $13-34$ & 14 & $30-37$ \\
Ma, number & $11-20$ & $14-29$ & 3 & $2-4$ \\
Mi, number & $2-5$ & $1-6$ & 3 & $2-3$ \\
DKs, number & $2-3$ & $2-3$ & Absent & Constantly 3 \\
Cirral rows left of ACR & Absent & Absent & - & Present \\
CG, shape & Spherical & Ellipsoidal & Absent \\
Data sources & Present study & Foissner et al. (2002) & Borror and Evans (1979) Foissner (1997) \\
\hline
\end{tabular}

All data are based on protargol-impregnated specimens.

AM, adoral membranelles; BC, buccal cirrus; FRC, frontotal-row cirri; ACR, amphisiellid median cirral row; LMC, left marginal cirri; RMC, right marginal cirri; Ma, macronuclei; Mi, micronuclei; DK, dorsal kineties; CG: cortical granules; -, uncertain data.

${ }^{a}$ Only one cell measured, ${ }^{b}$ Excluding frontal row cirri.

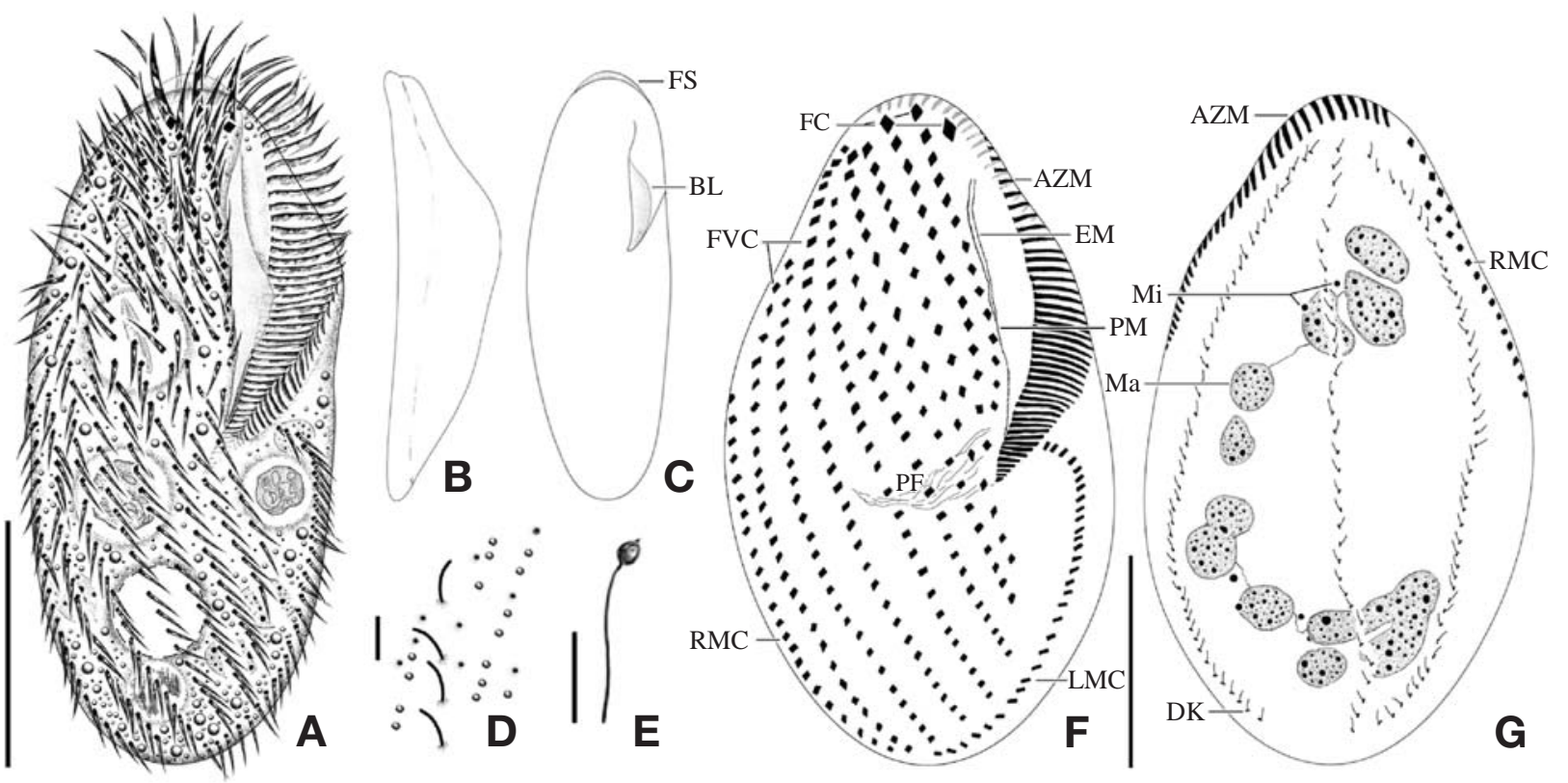

Fig. 4. Pseudokahliella marina from live $(A-E)$ and protargol impregnated specimens $(F, G)$. $A$, Ventral view of typical cell; $B$, Lateral view showing dorsoventrally flattened body shape; C, Elongated elliptical body shape; D, Arrangement of cortical granules and pore on dorsal sides; E, Ejected granules; F, Somatic and oral infraciliature of ventral side; G, Dorsal kineties and nuclear apparatus. AZM, adoral zone of membranelles; $\mathrm{BL}$, buccal lip; DK, dorsal kineties; EM, endoral membrane; FC, frontal cirri; FS, frontal scutum; FVC, frontoventral cirri; LMC, left marginal cirri; Ma, macronuclei; Mi, micronuclei; PF, pharyngeal fiber; PM, paroral membrane; RMC, right marginal cirri. Scale bars: $A, G=50 \mu \mathrm{m}, \mathrm{D}, \mathrm{E}=5 \mu \mathrm{m}$.

the frontoventral cirral row but leftmost cirrus distinctly enlarged and displaced leftwards (Figs. 4F, 6A, C). Usually 1113 frontoventral rows including left and right marginal row, arranged slightly obliquely backwards, each cirrus about 12 $\mu \mathrm{m}$ long (Figs. 4F, 6G). Left marginal row curved inwards in anterior portion, extending to posterior end, comprising 22-35 cirri (Figs. 4F, 6H). Right marginal row commencing from anterior one-third on dorsal side, comprising 28-40 cirri (Figs. 4G, 6F). Caudal and transverse cirri absent. Constantly three dorsal kineties with densely arranged cirri; bristle about 

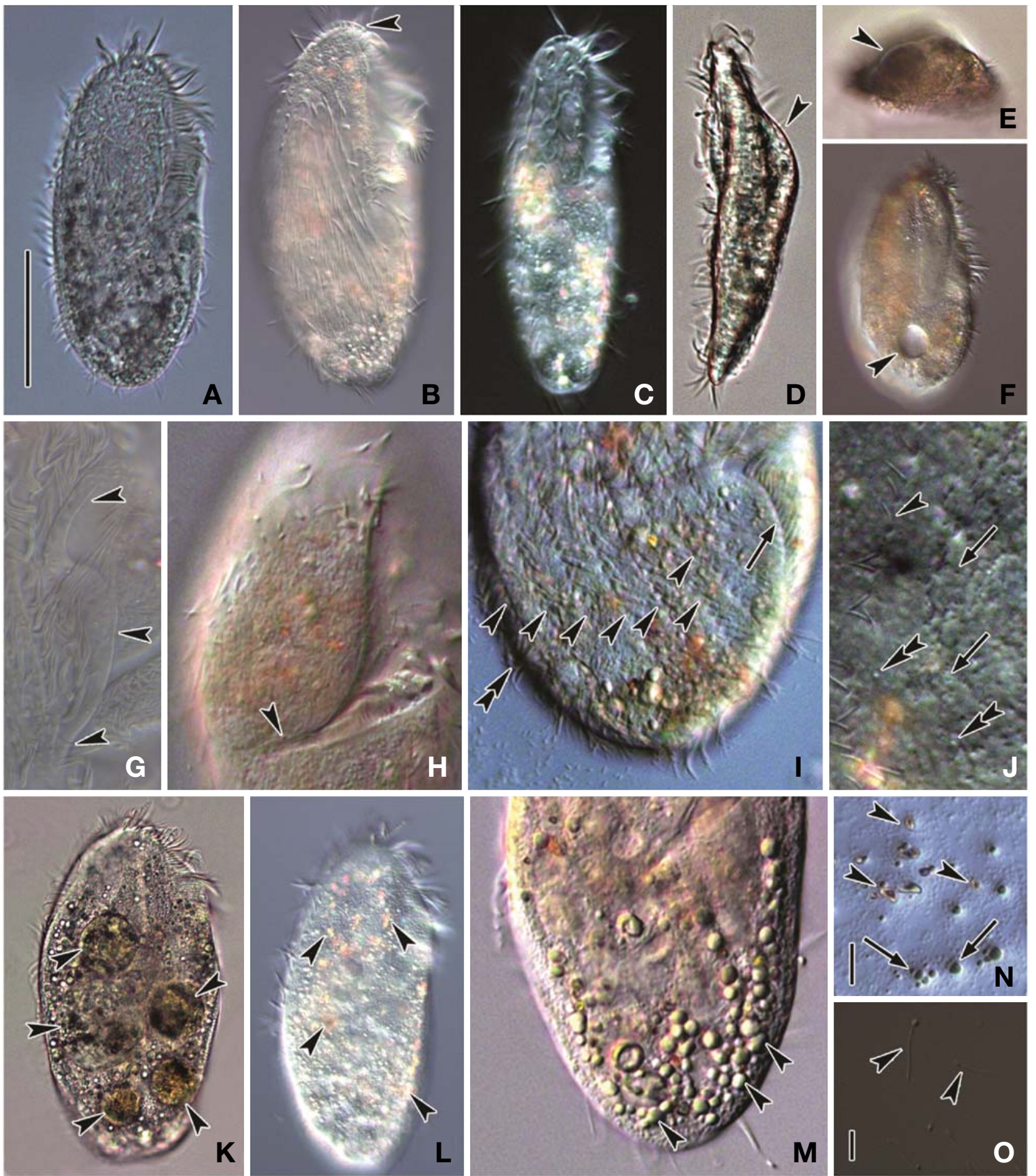

Fig. 5. Photomicrographs of Pseudokahliella marina from live specimens. A, Ventral view of typical cell; B, Somatic ciliature and frontal scutum (arrowhead); C, Elongated elliptical body shape; D, E, Ventral side flat while dorsal side convex (arrowheads) from lateral and apical view; F, Contractile vacuole (arrowhead); G, Prominent buccal lip (arrowheads); H, Cytopharynx (arrowhead); I, Left marginal cirri (arrow), frontoventral cirri (arrowheads) and right marginal cirri (double arrowhead); J, Dorsal bristle (arrowhead), cortical granules (double arrowheads) and pore (arrows); K, Food vacuoles (arrowheads); L, Crystals distributed in cell (arrowheads); M, Shining fat globules concentrated on posterior portion (arrowheads); N, Yellowish crystals (arrowheads) and greenish fat globules (arrows); O, Ejected granules (arrowheads). Scale bars: $\mathrm{A}=50 \mu \mathrm{m}, \mathrm{N}, \mathrm{O}=5 \mu \mathrm{m}$. 

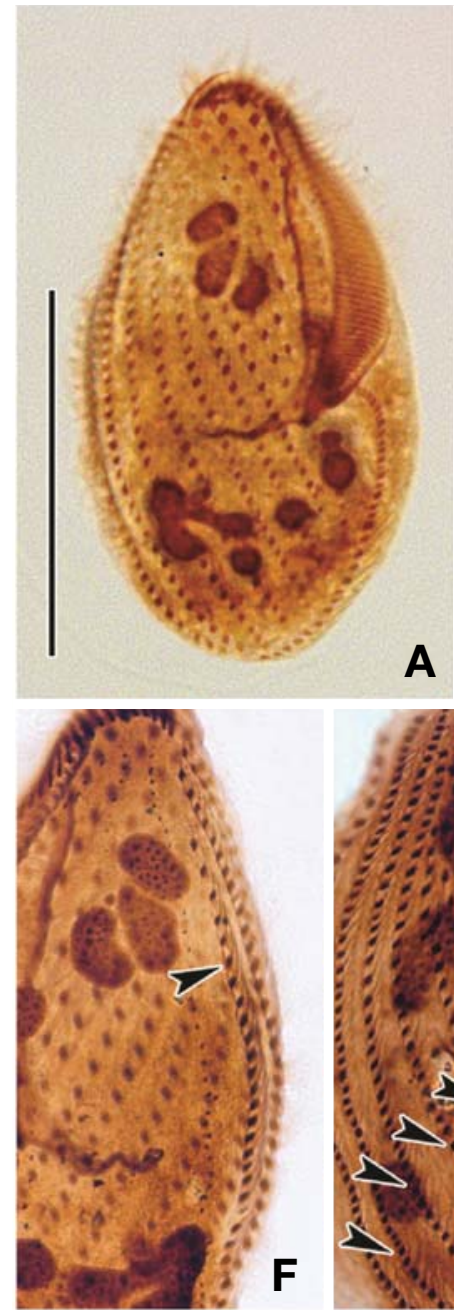

$\mathbf{F}$
A

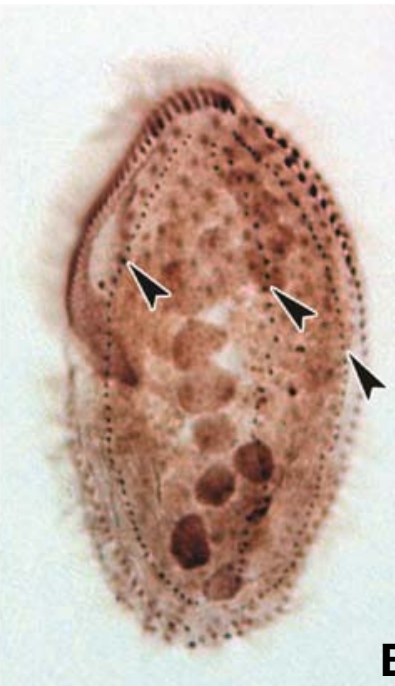

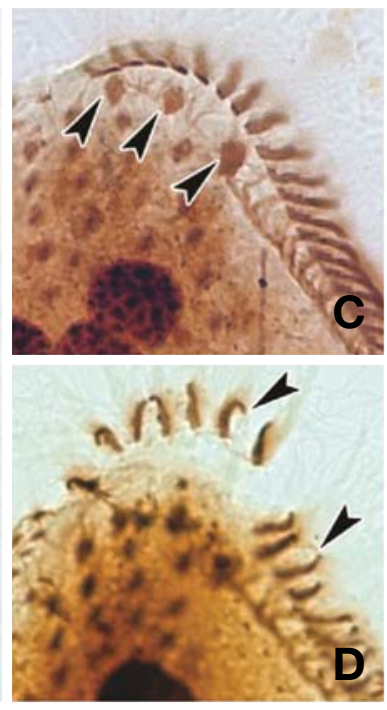

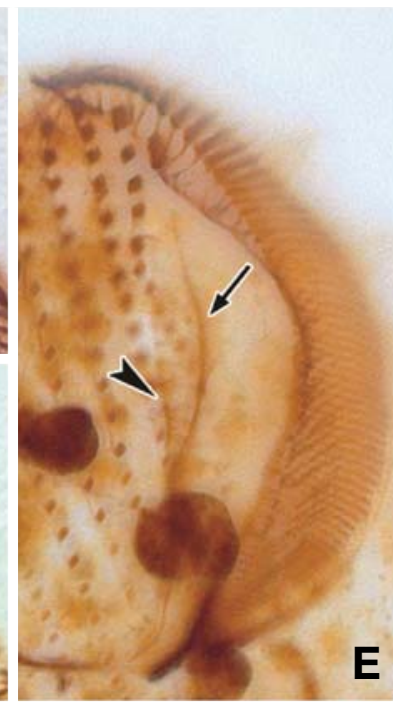

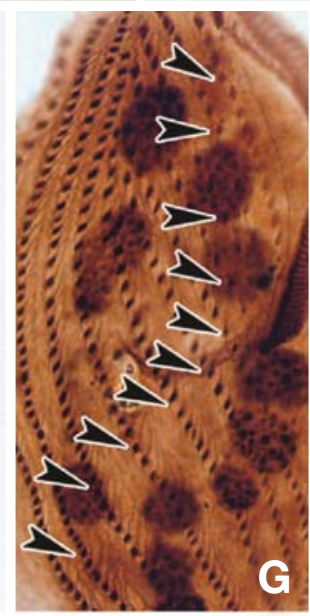
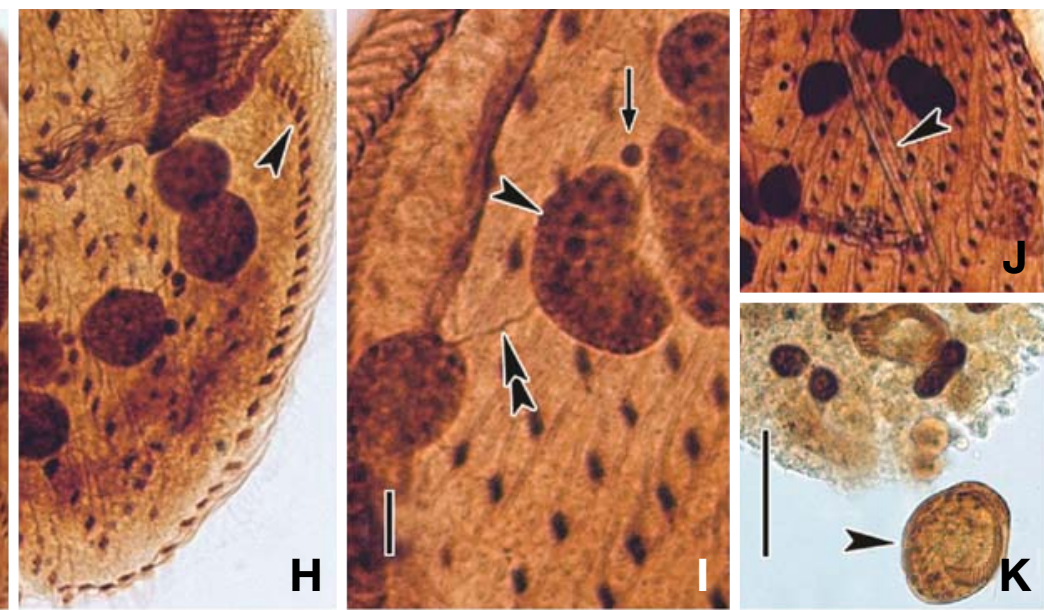

Fig. 6. Photomicrographs of Pseudokahliella marina from protargol-impregnated specimens. A, Ventral view of typical cell; $B$, Three dorsal kineties (arrowheads); C, Three frontal cirri (arrowheads); D, Hook-like shape of adoral membranelles (arrowheads); E, Paroral (arrowhead) and endoral (arrow) membranes; F, Right marginal cirral row on dorsal side (arrowhead); G, Frontoventral cirral rows (arrowheads); H, Left marginal row (arrowhead); I, Macronucleus (arrowhead) with thread-like structure (double arrowhead) and micronucleus (arrow); J, Ingested diatom (arrowhead); K, Ingested Euplotes sp. (arrowhead). Scale bars: $A=100 \mu \mathrm{m}, \mathrm{I}=5 \mu \mathrm{m}, \mathrm{K}=50$ $\mu \mathrm{m}$.

$5 \mu \mathrm{m}$ long (Figs. 4D, G, 5J, 6B).

Cortical granules: Cortical granules colourless about $1 \mu \mathrm{m}$ in diameter, sparsely arranged on dorsal surface and longitudinally between dorsal kineties but not detectable on ventral side, (Figs. 4D, 5J). Completely extruded forms of cortical granules, about $10 \mu \mathrm{m}$ long, club-like in shape, composed of spherical head and elongated stem (Figs. 4E, 5O).

Nuclear apparatus: Usually 8-11 macronuclear nodules, spherical to ellipsoid but rarely sausage-shaped, size 9-21 $\times$ 6-13 $\mu \mathrm{m}$, connected to each other by thread-like structures, forming C-shape in dorsal view. 7-9 micronuclei, sphericalshaped, near the macronuclei, and $2.0-2.5 \mu \mathrm{m}$ in diameter (Figs. 4G, 6I).
Distribution. Europe (France) and Asia (China, Korea [present study]).

Remarks. The genus Pseudokahliella is monotypic and established based on the original French population by Foissner et al. (1982). The characteristics of the Korean population of Pseudokahliella marina in this study agrees well with the original and subsequent descriptions in major aspects of morphology (Foissner et al., 1982; Hu and Song, 2003; Song et al., 2003, 2009). Generally, the Korean population slightly differs from the French population in the following characteristics: (1) number of adoral membranelles (ca. 56 vs. ca. 66); (2) length of adoral zone of membranelles (ca. $85 \mu \mathrm{m}$ vs. ca. $68 \mu \mathrm{m}$ ); (3) length of dorsal bristles (ca. $5 \mu \mathrm{m}$ vs. ca. 2 
Table 3. Morphometric data of Pseudokahliella marina

\begin{tabular}{lcccrrrrr}
\hline \multicolumn{1}{c}{ Characters } & Mean & Min & Max & Med & SD & SE & CV & $\mathrm{n}$ \\
\hline Body, length $(\mu \mathrm{m})$ & 161 & 109 & 208 & 161.1 & 22.50 & 5.03 & 14.01 & 20 \\
Body, width $(\mu \mathrm{m})$ & 82 & 62 & 105 & 80.1 & 11.80 & 2.64 & 14.40 & 20 \\
Body, length/width & 2.0 & 1.7 & 2.9 & 2.0 & 0.30 & 0.07 & 15.35 & 20 \\
AZM, length $(\mu \mathrm{m})$ & 85 & 68 & 100 & 87.1 & 10.09 & 2.26 & 11.88 & 20 \\
Body, length/AZM, length & 1.9 & 1.6 & 2.6 & 1.9 & 0.25 & 0.06 & 13.37 & 20 \\
AMs, number & 56 & 41 & 70 & 56.5 & 6.95 & 1.55 & 12.49 & 20 \\
Ma, number & 9 & 8 & 11 & 9.0 & 0.85 & 0.19 & 9.58 & 20 \\
Ma, length $(\mu \mathrm{m})$ & 15 & 9 & 21 & 14.8 & 4.58 & 1.02 & 30.05 & 20 \\
Ma, width $(\mu \mathrm{m})$ & 10 & 6 & 13 & 10.2 & 1.61 & 0.36 & 16.47 & 20 \\
FC, number & 3 & 3 & 3 & 3.0 & 0.00 & 0.00 & 0.00 & 18 \\
Frontoventral rows ${ }^{a}$, number & 12 & 11 & 13 & 12.0 & 0.66 & 0.16 & 5.46 & 17 \\
LMC, number & 29 & 22 & 35 & 29.0 & 2.96 & 0.66 & 10.31 & 20 \\
RMC, number & 34 & 28 & 40 & 34.0 & 3.86 & 1.00 & 11.41 & 15 \\
DKs, number & 3 & 3 & 3 & 3.0 & 0.00 & 0.00 & 0.00 & 11 \\
\hline
\end{tabular}

All data are based on protargol-impregnated specimens.

Min, minimum; Max, maximum; Med, median; SD, standard deviation; SE, standard error; CV, coefficient of variation in \%; $\mathrm{n}$, number of cells; $\mathrm{A}(\mathrm{Z}) \mathrm{M}$, adoral (zone of) membranelles; Ma, macronuclei; FC, frontal cirri; LMC, left marginal cirri; RMC, right marginal cirri; DK, dorsal kineties.

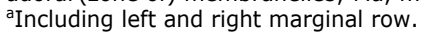

Table 4. Comparisons of different populations of Pseudokahliella marina

\begin{tabular}{|c|c|c|c|c|}
\hline Characters & P. marina & P. marina & P. marina ${ }^{\mathrm{a}}$ & \\
\hline Body, length $(\mu \mathrm{m})$ & $109-208$ & $112-182$ & $127-170$ & $102-194$ \\
\hline Body, width $(\mu \mathrm{m})$ & $62-105$ & $53-70$ & $72-110$ & $56-158$ \\
\hline AMs, number & $41-70$ & $56-84$ & $68-103$ & $52-104$ \\
\hline Ma, number & $8-11$ & $8-15$ & $10-20$ & $9-17$ \\
\hline $\mathrm{Ma}$, length $(\mu \mathrm{m})$ & $9-21$ & $7-22$ & $8-16$ & $13-29$ \\
\hline $\mathrm{Ma}$, width $(\mu \mathrm{m})$ & $6-13$ & $5.6-9$ & $6-11$ & $10-14$ \\
\hline $\mathrm{Mi}$, number & $7-9$ & $8-15$ & \multicolumn{2}{|c|}{ 4-8 } \\
\hline Mi diameter $(\mu \mathrm{m})$ & $2.0-2.5$ & Ca. 1.5 & \multicolumn{2}{|c|}{ Ca. 1.5} \\
\hline Cirral rows ${ }^{b}$, number & $11-13$ & $10-15$ & $8-10$ & $7-10$ \\
\hline LMC, number & $22-35$ & $23-37$ & $22-37$ & $28-38$ \\
\hline RMC, number & $28-40$ & $37-46$ & $24-47$ & $31-43$ \\
\hline $\mathrm{DB}$, length $(\mu \mathrm{m})$ & Ca. 5 & Ca. 5 & \multicolumn{2}{|c|}{$5-6$} \\
\hline UM, pattern & Two (separated) & Two (separated) & \multicolumn{2}{|c|}{ Single (double-rowed) } \\
\hline $\mathrm{CV}$, number & One & Two & \multicolumn{2}{|c|}{-} \\
\hline $\mathrm{CV}$, position & Behind mid-body & Mid-body and slightly ahead & \multicolumn{2}{|c|}{-} \\
\hline $\mathrm{CG}$, diameter $(\mu \mathrm{m})$ & Ca. 1 & Ca. 1.5 & \multicolumn{2}{|c|}{ Ca. 1} \\
\hline Ejected CG, shape & Club-shape & - & \multicolumn{2}{|c|}{ Spindle } \\
\hline Ejected CG, length $(\mu \mathrm{m})$ & Ca. 11 & - & \multicolumn{2}{|c|}{$5-7$} \\
\hline Data sources & Present study & Foissner et al. (1982) & \multicolumn{2}{|c|}{ Hu and Song (2003) } \\
\hline
\end{tabular}

All data are based on protargol-impregnated specimens.

$\mathrm{AM}$, adoral membranelles; Ma, macronuclei; Mi, micronuclei; LMC, left marginal cirri; RMC, right marginal cirri; DB, dorsal bristles; UM, undulating membrane; CV, coefficient of variation in \%; CG, cortical granules; - , uncertain data.

${ }^{a}$ Two populations collected in 1996 and in 2000, bincluding left and right marginal row.

$\mu \mathrm{m})$; (4) diameter of micronucleus $(2.0-2.5 \mu \mathrm{m}$ vs. ca. 1.5 $\mu \mathrm{m}$ ); (5) diameter of cortical granules (ca. $1 \mu \mathrm{m}$ vs. ca. 1.5 $\mu \mathrm{m}$ ); (6) number of contractile vacuoles (one vs. two); (7) position of contractile vacuoles (behind mid-body vs. midbody and slightly ahead) (Foissner et al., 1982; Berger, 2011). In addition, the following features are minor differences between the Korean and Chinese populations: (1) pattern of undulating membranes (two [separated] vs. single [doublerowed]); (2) number of cirral rows including both marginal rows (11-13 vs. 7-10); (3) shape and length of ejected cortical granules (club-like, ca. $11 \mu \mathrm{m}$ vs. spindle, 5-7 $\mu \mathrm{m})(\mathrm{Hu}$ and Song, 2003; Berger, 2011) (Table 4).

\section{ACKNOWLEDGMENTS}

This study was supported by the grant from the Korean Institute of Ocean Science and Technology (PE98746), the 
National Research Foundation of Korea Grant funded by the Korea Government (No. 2009-0077263), and "The Survey of Korean Indigenous Species" supported by the National Institute of Biological Resources (NIBR) of Korea.

\section{REFERENCES}

Berger H, 1999. Monograph of the Oxytrichidae (Ciliophora, Hypotrichia). Monographiae Biologicae, 78:1-1079.

Berger H, 2001. Catalogue of ciliate names 1. Hypotrichs. Verlag Helmut Berger, Salzburg, pp. 1-206.

Berger H, 2006. Monograph of the Urostyloidea (Ciliophora, Hypotricha). Monographiae Biologicae, 85:1-1303.

Berger H, 2008. Monograph of the Amphisiellidae and Trachelostylidae (Ciliophora, Hypotricha). Monographiae Biologicae, 88:1-737.

Berger H, 2011. Monograph of the Gonostomatidae and Kahliellidae (Ciliophora, Hypotricha). Monographie Biologicae, 90:1-741.

Berger H, Foissner W, Adam H, 1985. Morphological variation and comparative analysis of morphogenesis in Parakahliella macrostoma (Foissner, 1982) nov. gen. and Histriculus muscorum (Kahl, 1932), (Ciliophora, Hypotrichida). Protistologica, 21:295-311.

Borror AC, Evans FR, 1979. Cladotricha and phylogeny in the suborder Stichotrichina (Ciliophora, Hypotrichida). Journal of Protozoology, 26:51-55.

Corliss JO, 1979. The ciliated protozoa: characterization, classification and guide to the literature. Pergamon Press, London and New York, pp. 1-455.

Fauré-Fremiet E, 1961. Remarques sur la morphologie comparée et la systématique des ciliata Hypotrichida. Comptes Rendus Hebdomadaires des Séances de l'Académie des Sciences, Paris, 252:3515-3519.

Foissner W, 1992. Protargol methods. In: Protocols in protozoology (Eds., Lee JJ, Soldo AT). The Society of Protozoologists, Lawrence, KS, p. C6.1-C6.8.

Foissner W, 1997. Soil ciliates (Protozoa: Ciliophora) from evergreen rain forests of Australia, South America and Costa Rica: diversity and description of new species. Biology and
Fertility of Soils, 25:317-339.

Foissner W, Adam H, Foissner I, 1982. Morphologie und Infraciliatur von Bryometopus pseudochilodon Kahl, 1932, Balantidioides dragescoi nov. spec. und Kahliella marina nov. spec. und Revision des Genus Balantioides Penard, 1930 (Protozoa, Ciliophora). Protistologica, 18:211-225.

Foissner W, Agatha S, Berger H, 2002. Soil ciliates (Protozoa, Ciliophora) from Namibia (Southwest Africa), with emphasis on two contrasting environments, the Etosha region and the Namib Desert. Denisia, 5:1-1459.

Hu X, Song W, 2003. Redescription of the morphology and divisional morphogenesis of the marine hypotrich Pseudokahliella marina (Foissner et al., 1982) from scallop-culture water of North China. Journal of Natural History, 37:20332043.

Lee JJ, Small EB, Lynn DH, Bovee EC, 1985. Some techniques for collecting, cultivating and observing protozoa. In: An illustrated guide to the protozoa (Eds., Lee JJ, Hunter SH, Bovee EC). Society of Protozoologists, Lawrence, KS, pp. 1-7.

Lynn DH, 2008. The ciliated protozoa: characterization, classification, and guide to the literature. 3rd ed. Springer Publishers, New York, pp. 1-605.

Lynn DH, Small EB, 2002. Phylum Ciliophora. In: An illustrated guide to the protozoa: organisms traditionally referred to as protozoa, or newly discovered groups. 2nd ed. Vol. 1 (Eds., Lee JJK, Leedale GF, Bradbury PC). Society of Protozoologists, Lawrence, KS, pp. 371-656.

Shin MK, 1994. Systematics of Korean hypotrichs (Ciliophora, Polyhymenophora, Hypotrichida) and molecular evolution of hypotrichs. PhD dissertation, Seoul National University, Seoul, Korea, pp. 1-270.

Song W, Warren A, Hu X, 2009. Free-living ciliates in the Bohai and Yellow Seas, China. Science Press, Beijing, pp. 1-518.

Song W, Zhao Y, Xu K, Hu X, Gong J, 2003. Pathogenic protozoa in mariculture. Science Press, Beijing, pp. 1-483.

Wilbert N, 1975. Eine Verbesserte Technik der Protargolimpragnation für Ciliaten. Mikrokosmos, 64:171-179.

Received April 20, 2012 Revised June 30, 2012 Accepted July 14, 2012 